\title{
Characterization of clay deposits in the Adrar region, with a view to their valorization in the building materials industry
}

\section{Caractérisation des gisements d'argiles de la région d'Adrar, en vue de leur valorisation dans l'industrie des matériaux de construction}

\author{
ABBOU Mohammed ${ }^{1}$, MOULAY OMAR Hassan ${ }^{1}$, SEMCHA Abdélaziz ${ }^{1}$, KAZI-AOUAL Fatiha ${ }^{2}$ \\ 1 Ahmed Draia University of Adrar, Science and Technology, Adrar, Algeria \\ 2 National Polytechnic School of Oran, Civil engineering, Oran, Algeria
}

\begin{abstract}
In the context of sustainable local development of the Adrar region, one of the largest regions in the Algerian Sahara. The search for local useful substances has been initiated by the Algerian state to cover the need for building materials in the construction industry. However, from a geological point of view, the Adrar zone is located in the extension of the primary chain of the Ougarta which separates two sedimentary basins of Reggane and Timimoun, as well as the basin of Sbâa. In this context, an experimental study is focused on the characterization of clay deposits, with a view to their valorization in the construction materials industry (ceramics) sector, with the aim of contributing to the use of local materials.
\end{abstract}

Keywords: clay; Ceramic; building materials; Adrar; development.

\begin{abstract}
Résumé. Dans le cadre du développement local durable de la région d'Adrar, l'une des plus vastes régions dans le Sahara algérien, la recherche de substances utiles locales a été entamée par l'état algérien, cela pour répondre au besoin en matériaux de construction dans le BTP. Cependant du point de vue géologique, la zone d'Adrar est située dans le prolongement de la chaine primaire de l'Ougarta qui sépare deux bassins sédimentaires : celui de Réggane et celui de Timimoun, ainsi que la cuvette de Sbâa. Dans ce contexte, une étude expérimentale est focalisée sur la caractérisation des gisements argileux, en vue de leur valorisation dans le secteur de l'industrie des matériaux de construction (céramique), ayant pour objet de contribuer à l'utilisation des matériaux locaux.
\end{abstract}

Mots-Clés : argile ; céramique ; matériau de construction ; Adrar ; développement.

\section{Introduction}

Durant ces dernières années le développement de la construction des logements au Sud Algérien, telle que la région d'Adrar, l'une des plus vastes régions dans le Sud algérien a engendré un besoin énorme en matériaux de construction conventionnels. Des efforts ont été orientés vers le développement de nouvelles méthodes de construction en utilisant des matériaux locaux afin d'apporter une réponse adéquate à cette crise.

Dans ce travail, nous présentons la localisation géologique de la région du Touat (Adrar). Par la suite nous ferons une présentation de l'étude expérimentale qui englobera l'identification de l'argile de la région d'Adrar et au préalable une présentation des différentes méthodes utilisées pour identifier notre argile.
Par ailleurs cet article regroupe les caractéristiques céramiques préliminaires de l'argile de la région d'Adrar (Sbaa).

Finalement, nous avons ciblé l'orientation de notre argile dans le secteur de fabrication des produits céramique (Brique de terre cuite).

\section{Contexte géologique du site}

La région d'étude se situe dans l'Ouest-Sud-Ouest du Sahara algérien au niveau de la plate-forme saharienne (Fig.1). Des ergs (massifs de dunes de sable) et des regs (plateaux caillouteux) constituent les paysages de la zone 
étudiée. Seuls les monts d'Ougarta présentent des reliefs significatifs.

Par ailleurs, les affleurements se rencontrent dans le lit des oueds limités par des hamadas plateaux [1].

Dans ce contexte géologique particulier la région d'Adrar dispose d'une grande réserve de dépôts sédimentaires de nature argileuse ainsi que d'un lit de sable de dune d'épaisseur énorme [2].

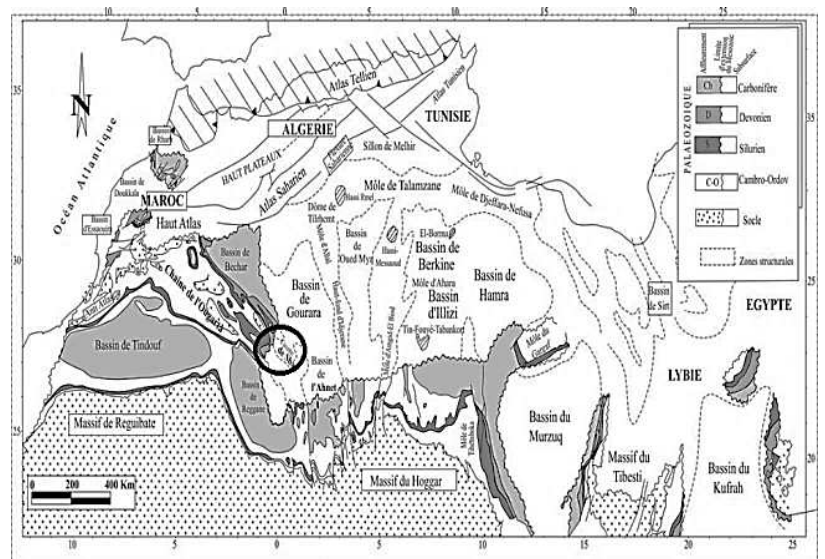

Fig. 1 Carte des principaux éléments morpho-structuraux de la plate-forme saharienne (modifiée d'après Boote et al., 1998)[1].

\section{Matériau et méthodes}

\subsection{Matériau}

Les sols sont différents d'une région à une autre, pour cela, une identification de leurs caractéristiques s'impose. L'argile étudiée se localise dans le crétacé inférieur communément appelé continentale intercalaire. Dans la première partie du secteur des sédiments du crétacé inférieur sont recouverts par une épaisse couche de dépôts récents (sable, limon, débris de grés, galets de quartz et localement d'anhydrite).

Le site est situé à quelques kilomètres au nord de la ville d'Adrar (Est de la RN6). Le matériau a une couleur rouge.

\subsection{Méthodes}

Dans notre étude nous devons identifier le gisement d'argile de la région d'Adrar (Sbâa). La distribution granulométrique a été déterminée par deux méthodes complémentaires, le tamisage par voie humide et une analyse par sédimentation, respectivement selon les normes XP P94-041 [4] et NF P 94-057 [5]. Les propriétés plastiques de la fraction fine, particules de taille inférieure à $400 \mu \mathrm{m}$, ont été mesurées comme il est défini dans la NF P94-051 [6]. La masse volumique des particules solides (Gs) a été mesurée en utilisant un pycnomètre NF P 94-054 [7].

Les analyses des compositions chimiques sommaires sont effectuées au laboratoire LTPO à Béchar (Algérie).
Les essais réduits sur l'argile pour déterminer les caractéristiques céramiques ont été réalisés au niveau du laboratoire CETIM à Boumerdès (Algérie).

\subsection{Résultats et discussions}

\subsubsection{Caractéristiques géotechniques et chimiques}

Selon les classifications LCPC, cette argile est très plastique (At), et d'activité normale $(\mathrm{Ac}=\mathrm{Ip} / \%<2 \mu \mathrm{m})$. La surface spécifique totale déduite de l'essai au bleu de méthylène par la formule de Tran Ngoc Lan (1977) [3], est de $230,23 \mathrm{~m}^{2} / \mathrm{g}$. Le tableau 1 résume les résultats d'identification de l'argile étudiée.

Tableau 1. Propriétés de l'argile utilisée.

\begin{tabular}{|c|c|c|}
\hline Paramètres & Symbole & Valeurs \\
\hline $\begin{array}{c}\text { Poids volumique des } \\
\text { grains solides }\end{array}$ & $\gamma \mathrm{s}\left(\mathrm{g} / \mathrm{cm}^{3}\right)$ & 2,60 \\
\hline Limite de liquidité & $\omega_{\mathrm{L}}(\%)$ & 81 \\
\hline Limite de plasticité & $\omega_{\mathrm{p}}(\%)$ & 34 \\
\hline Indice de plasticité & $\mathrm{I}_{\mathrm{P}}$ & 47 \\
\hline Limite de retrait & $\omega_{\mathrm{R}}(\%)$ & 19,34 \\
\hline Sable & $\mathrm{F} 1(\%)$ & 10 \\
\hline Limon & $\mathrm{F} 2(\%)$ & 54 \\
\hline Argile & $\mathrm{F} 3(\%)$ & 36 \\
\hline Activité des argiles & $\mathrm{A}_{\mathrm{C}}$ & 1,30 \\
\hline $\begin{array}{c}\text { La valeur de bleu de } \\
\text { méthylène }\end{array}$ & $\mathrm{VB}(\%)$ & 11 \\
\hline
\end{tabular}

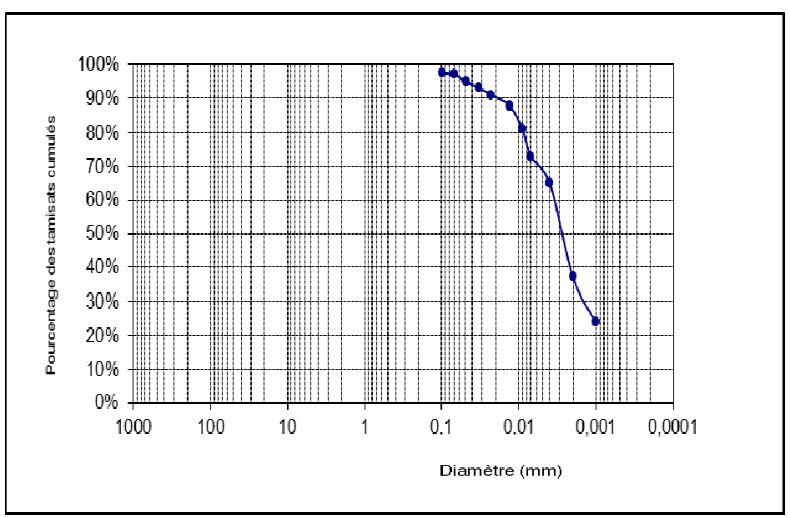

Fig. 2. Courbe granulométrique de l'argile de Sbâa

Suite aux résultats obtenus nous pouvons dire que l'argile est un matériau silico-calcaire faiblement gypseux. Le tableau 3 regroupe les valeurs de composition chimique sommaire de l'argile étudiée.

Tableau 3. Composition chimique sommaire de l'argile.

\begin{tabular}{|c|c|}
\hline Paramètres & Valeurs (\%) \\
\hline $\mathrm{SO}_{4}{ }^{2-}$ & 0,41 \\
\hline $\mathrm{CaCO}_{3}$ & 3.6 \\
\hline $\mathrm{Cl}^{-}$ & 0,14 \\
\hline
\end{tabular}




\begin{tabular}{|l|l|}
\hline $\begin{array}{l}\text { Insolubles }\left(\mathrm{SiO}_{2}-\mathrm{Al} 2 \mathrm{O} 3-\right. \\
\mathrm{Fe} 2 \mathrm{O} 3-\mathrm{CaO}-\mathrm{MgO})\end{array}$ & 95,92 \\
& \\
\hline
\end{tabular}

\subsubsection{Caractéristiques céramiques}

\section{- Propriétés de façonnage et de séchage}

Le tableau 4 ci-après résume les résultats obtenus des essais de façonnage et de séchage.

Tableau 4. Propriétés de façonnage et de séchage de l'argile d'Adrar.

\begin{tabular}{|c|c|c|c|c|}
\hline \multicolumn{5}{|c|}{ Caractéristiques de séchage } \\
\hline $\begin{array}{c}\text { Régime } \\
\text { de } \\
\text { séchage }\end{array}$ & $\begin{array}{c}\text { Humidité } \\
\text { de } \\
\text { façonnag } \\
\text { e (\%) }\end{array}$ & $\begin{array}{c}\text { Retrait } \\
\text { sur sec } \\
(\%)\end{array}$ & $\begin{array}{c}\text { Résistance } \\
\text { mécanique } \\
\text { en }\left(\mathbf{k g} / \mathbf{c m}^{2}\right)\end{array}$ & coloration \\
\hline Lent & 23,1 & 7,8 & 50,14 & Rouge \\
\hline
\end{tabular}

L'échantillon d'argile d'Adrar (Sbaa) présente un bon aspect physique. Après séchage lent il ressort ce qui suit :

\section{a)Sur le plan céramique :}

-Retrait sur sec et humidité de façonnage sont acceptables pour l'argile de Sbâa.

-Résistance mécanique sur sec est admissible.

$$
\begin{aligned}
& \text { b) Sur le plan aspect physique : } \\
& \text { - Bon aspect physique. } \\
& \text { - Coloration : Rouge. }
\end{aligned}
$$

\section{- Propriétés de cuisson à $900^{\circ} \mathrm{C}$}

Les paramètres de cuisson de l'argile sont regroupés dans le tableau. 5

Tableau 5. Propriétés de cuisson à $900^{\circ} \mathrm{C}$ de l'argile étudiée.

\begin{tabular}{|l|l|c|c|c|}
\hline $\begin{array}{l}\text { Perte } \\
\text { au } \\
\text { Feu }\end{array}$ & $\begin{array}{l}\text { Absorption } \\
\text { d'eau } \\
\text { en } \mathbf{( \% )}\end{array}$ & $\begin{array}{c}\text { Retrait } \\
\text { sur cuit } \\
\mathbf{( \% )}\end{array}$ & $\begin{array}{c}\text { Poids } \\
\text { volumique } \\
\left(\mathbf{g} / \mathbf{d m}^{\mathbf{3}}\right)\end{array}$ & coloration \\
\hline 10,2 & 6,8 & 2,4 & 1799 & Rouge \\
\hline
\end{tabular}

Le tesson de l'échantillon cuit à $900^{\circ} \mathrm{C}$ présente les caractéristiques suivantes :

a) Sur le plan céramique

- Le retrait total : Trop élevé

- Absorption : Acceptable

b) Sur le plan aspect physique

-Coloration : Rouge.
Dans le contexte géologique, la région d'Adrar est située dans la cuvette limitée par deux grandes bassins de sédimentaire le bassin de Timimoun et le bassin de Reggane. Devant cette formation la région dispose d'une grande réserve de sédiments de nature argileuse continentale intercalaire.

Cette étude a permis :

- d'utiliser des matériaux locaux, et d'identifier les caractéristiques physico-mécanique et chimique de l'argile de la région d'Adrar dans le but de l'utiliser comme matières de base pour la fabrication des produits céramiques tels que brique, tuile et carreaux.

- de présenter les caractéristiques géotechniques, ainsi que la composition chimique sommaire de l'argile d'Adrar.

Nous avons mis en évidence à travers cette étude les caractéristiques céramiques notamment les propriétés de façonnage et de séchage puis les propriétés de cuisson à $900^{\circ} \mathrm{C}$ qui sont conformes aux recommandations des exigences céramiques.

Enfin, à travers les résultats de cette étude, nous pouvons avancer que :

-L'argile d'Adrar (Sbâa) peut faire l'objet de matière de base pour la fabrication de briques de terre cuite.

-Moyennant un ajout correcteur du retrait total excessif, il reste cependant à étudier la composition minéralogique et chimique détaillées, cela pour bien orienter l'argile d'Adrar (Sbâa) vers l'industrie des produits céramiques.

\section{Références}

1. Boote D.R.D., Clark-Lowes D.D. et Traut M.W. Paleozoïc petroleum systems of North Africa. In : Petroleum Geology of North Africa, éds. D.S. Macgregor, R.T.J. Moody and D. D. ClarkLowes, Geol. Soc. Spec. Pub., 133, 7-68(1998).

2. Gautier E.-F., Larnaude Marcel. L'oued Saoura. In: Annales de Géographie, t. 30, n¹63, pp. 5059, 1921.

3. Tran Ngoc Lan. "Nouvel essai d'identification des sols : l'essai au bleu de méthylène", Bulletin de Liaison des Ponts et Chaussées, 88, pp. 136137, (Mars - Avril 1977).

4. AFNOR. XP P 94-041. Sols : reconnaissance et essais -Identification granulométrique Méthode de tamisage par voie humide. AFNOR, (1995).

5. AFNOR. NF P 94-057. Sols : reconnaissance et essais - Analyse granulométrique des sols Méthode par sédimentation. AFNOR, (1992).

6. AFNOR. NF P 94-051; Sols : reconnaissance et essais - Détermination des limites d'Atterberg Limite de liquidité à la coupelle - Limite de plasticité au rouleau. AFNOR, (1993).

7. AFNOR. NF P 94-054; Sols : reconnaissance et essais - Détermination de la masse volumique des particules solide des sols - Méthodes du pycnomètre à l'eau. AFNOR, (1991).

\section{Conclusion}

